\title{
Molecular epidemiology of rhinovirus among hospitalised patients, Singapore
}

\author{
Chun Kiat Lee, ${ }^{1}$ Erik Wei Jun Low, ${ }^{2}$ Christian Benjamin George Highfield, ${ }^{2}$ Hong Kai Lee, ${ }^{1}$ \\ Paul Anantharajah Tambyah, ${ }^{3}$ Tze Ping Loh, ${ }^{1}$ Evelyn Siew-Chuan Koay ${ }^{1,4}$ \\ ${ }^{1}$ Department of Laboratory Medicine, National University Hospital; ${ }^{2}$ Ngee Ann Polytechnic; \\ ${ }^{3}$ Department of Medicine, Yong Loo Lin School of Medicine, National University of Singapore; \\ ${ }^{4}$ Department of Pathology, Yong Loo Lin School of Medicine, National University of Singapore
}

\section{Summary}

Human rhinovirus (HRV) is the most prevalent respiratory etiological agent in the world. Over 100 genotypes have been characterised using molecular genotyping techniques. Here, we characterised the molecular epidemiology of the circulating rhinoviruses among hospitalised patients in Singapore by sequencing 134 rhinovirus-positive respiratory specimens that were collected in the period between 2013 and 2015. Each sequence was assigned a genogroup and a genotype using the Enterovirus Genotyping Tool Version 0.1 and phylogenetic reconstruction, respectively. In this study, HRV-A $(n=88)$ and HRV-C $(\mathrm{n}=38)$ were identified as the dominant genogroups in Singapore. HRV-A28 ( $n=7)$ was the dominant genotype in HRV-A while both HRV$\mathrm{C} 2(\mathrm{n}=8)$ and HRV-C11 $(\mathrm{n}=8)$ were the dominant genotypes in HRV-C. HRV-B was observed to have the lowest number of positive detections in our study population $(n=8)$. The result is interesting as another group had previously found HRV-B to be the second most common genogroup in Singapore after HRV-A.

Correspondence: Chun Kiat Lee, Department of Laboratory Medicine, National University Hospital, 5 Lower Kent Ridge Road, 119074, Singapore. Tel.: +65.6772 .4175 - Fax: +65.6772 .4407$

E-mail: chunkiatlee1983@gmail.com

Key words: Respiratory tract infections; rhinovirus; genotyping; RT-PCR; epidemiology; molecular diagnostics

Contributions: the authors contributed equally.

Conflict of interest: the authors declare no potential conflict of interest.

Received for publication: 16 August 2016.

Accepted for publication: 18 September 2016.

(C) Copyright C.K. Lee et al., 2016

Licensee PAGEPress, Italy

Microbiologia Medica 2016; 31:6233

doi:10.4081/mm.2016.6233

This article is distributed under the terms of the Creative Commons Attribution Noncommercial License (by-nc 4.0) which permits any noncommercial use, distribution, and reproduction in any medium, provided the original author(s) and source are credited.

\section{Introduction}

The human rhinoviruses (HRVs, family Picornaviridae, genus Enterovirus) are positive-sense, single-stranded-RNA viruses and are responsible for more than $50 \%$ of all common cold incidences (2). HRV exhibits high genetic diversity and over 100 genotypes have been categorised. Previously, HRVs were serotyped using serotype-specific antisera to differentiate between HRV-A and HRVB. Recently, a hitherto unreported genogroup, HRV-C, has been identified using molecular genotyping techniques. The traditional serotyping method is unable to detect this genogroup as it cannot be cultured (1).

To the best of our knowledge, there has only been one Singaporebased study that examined the molecular epidemiology of HRVs (6). The study was conducted in the period between 2005 and 2007 and the study population involved hospitalised children only. In the present study, we aimed to characterise the more recent molecular epidemiology of the circulating HRVs among hospitalised patients in Singapore. One-hundred and thirty-four HRV-positive specimens, collected in the period between 2013 and 2015, were included in the study. The specimens were genotyped using a previously described method (3).

\section{Materials and Methods}

This study received local institutional ethics approval (from the National Healthcare Group Domain-Specific Review Board A, reference number: 2016/00044). The study was carried out on 134 clinical respiratory specimens that were submitted to the Molecular Diagnosis Centre, Singapore National University Hospital, for respiratory virus detection in the period between January 2013 and June 2015. These clinical specimens were archived specimens that were previously identified as HRV positive by a laboratory-developed multiplex RT-PCR respiratory viral panel. In the current study, a set of primers was used to amplify a 390-bp region within the 5'UTR as previously described (3). The RT-PCR products were then purified with the QIAGEN QIAquick gel extraction kit (Qiagen, Hilden, Germany) and sequenced bi-directionally using the Applied Biosystems 3130xl genetic analyzer (Thermo Fisher Scientific, Wohlen, Switzerland). Assembly of the sequences was performed using the Assign ${ }^{\text {TM }}$ ATF software (Conexio Genomics, Fremantle, Australia). A web-based typing website, Enterovirus Genotyping Tool Version 0.1 was used for genogroup assignment (4). Genotype assignment was conducted based on phylogenetic inference using minimum-evolution subtreepruning-regrafting and maximum-likelihood nearest-neighbour 
interchanges with 1000 bootstrapped replicates. The evolutionary distances were computed based on the general time reversible nucleotide substitution model with FastTree 2 (5). One hundred and fifty-six HRV complete genomes were obtained from GenBank to serve as reference genotypes in the phylogenetic analysis.

\section{Results}

All of the 134 specimens were successfully sequenced. The phylogenetic reconstruction was able to correctly classify the 156 GenBank sequences according to their respective genotypes. The 134 clinically isolated HRV specimens were also fully assigned a genotype based on the phylogenetic analysis. A total of 60 unique genotypes were identified. Of these unique genotypes, $66.7 \%(40 / 60)$ belonged to HRV-A, $11.7 \%$ (7/60) belonged to HRV-B, and $21.7 \%$ (13/60) belonged to HRVC. For genogroups, 65.7\% (88/134) were HRV-A, 6.0\% (8/134) were HRV-B, and $28.3 \%$ (38/134) were HRV-C.

\section{Discussions and Conclusions}

In summary, we described the use of a partial 5UTR region of the HRV genome for accurate typing of 134 rhinovirus-positive specimens that were collected from hospitalised patients over a period of three years. In this study, HRV-A and HRV-C were identified as the dominant genogroups in Singapore. HRV-B has the lowest number of positive detections in our study population. This is interesting as another group had previously found HRV-B to be the second most common genogroup in Singapore (6). This discrepancy may be attributed to the different recruitment criteria of the study population (hospitalised children $v s$. hospitalised patients of all age groups) and sampling periods (20052007 vs. 2013-2015). HRV-A28 ( $\mathrm{n}=7)$ was the dominant genotype in
HRV-A while both HRV-C2 ( $n=8)$ and HRV-C11 $(n=8)$ were the dominant genotypes in HRV-C. None of the HRV-B genotypes appears to be dominant due to the low prevalence of HRV-B.

There have been several reports on the association of HRV-C infection with asthma attacks, wheezing occurrence, and severe pneumonia (6-8). Therefore, the ability to provide genotype information for the HRV strain that is infecting a patient may present an advantage for the prevention and control of HRV-associated acute respiratory diseases.

\section{References}

1. Bochkov YA, Gern JE. Clinical and molecular features of human rhinovirus C. Microbes Infect 2012;14:485-94.

2. Jacobs SE, Lamson DM, St George K, et al. Human rhinoviruses. Clin Microbiol Rev 2013;26:135-62.

3. Kiang D, Kalra I, Yagi S, et al. Assay for 5 ' noncoding region analysis of all human rhinovirus prototype strains. J Clin Microbiol 2008;46:3736-45.

4. Kroneman A, Vennema H, Deforche K, et al. An automated genotyping tool for enteroviruses and noroviruses. J Clin Virol 2011;51:121-5.

5. Price MN, Dehal PS, Arkin AP. FastTree 2: approximately maximumlikelihood trees for large alignments. PLoS One 2010;5: e9490.

6. Tan BH, Loo LH, Lim EA, et al. Human rhinovirus group C in hospitalised children, Singapore. Emerg Infect Dis 2009;15:1318-20.

7. Lau SK, Yip CC, Tsoi HW, et al. Clinical features and complete genome characterization of a distinct human rhinovirus (HRV) genetic cluster, probably representing a previously undetected HRV species, HRV-C, associated with acute respiratory illness in children. J Clin Microbiol 2007;45:3655-64.

8. McErlean P, Shackelton LA, Lambert SB, et al. Characterisation of a newly identified human rhinovirus, HRV-QPM, discovered in infants with bronchiolitis. J Clin Virol 2007;39:67-75. 